\title{
O Uso de um Laboratório Virtual de Matemática no Processo de Ensino-aprendizagem
}

\author{
Daniela Rodrigues Ribas \\ Programa de Pós-Graduação em Informática na Educação \\ Universidade Federal do Rio Grande do Sul (PPGIE/UFRGS) \\ Faculdade de Matemática \\ Pontifícia Universidade Católica do Rio Grande do Sul (FAMAT/PUCRS) \\ daniela.ribas@pucrs.br \\ Dante Augusto Couto Barone \\ Programa de Pós-Graduação em Informática na Educação \\ Universidade Federal do Rio Grande do Sul (PPGIE/UFRGS) \\ barone@inf.ufrgs.br \\ Marcus Vinícius de Azevedo Basso \\ Instituto de Matemática \\ Universidade Federal do Rio Grande do Sul (IMA/UFRGS) \\ mbasso@ufrgs.br
}

Resumo: Solucionar problemas é algo que fazemos diariamente, mesmo que muitas vezes nem se perceba. Quanto mais trabalharmos tal habilidade, mais facilmente levantaremos hipóteses e chegaremos a possíveis soluções. Materiais instrucionais e desafios lógicos podem ser de grande importância nessa prática. Este artigo apresenta uma reflexão sobre o ensino-aprendizagem de matemática, o uso de materiais instrucionais e levanta o uso das tecnologias computacionais em tal processo. Aborda a resolução de problemas, dando ênfase aos desafios lógicos, e então apresenta um protótipo de laboratório virtual de matemática para ser trabalhado com tal proposta.

Palavras-chave: ensino de matemática, resolução de problemas, desafios lógicos, recursos computacionais no ensino de matemática.

\begin{abstract}
Solving problems is something we do every day, even if not often see. The more work that ability, easier we have hypotheses and we can arrive possible solutions. Instructional materials and logic puzzle can be of great importance in this practice. This paper presents a reflection on the teaching-learning of mathematics, the use of instructional materials and the use of computer technology in this process. Talk about the resolution of problems, emphasizing the logic puzzle, and then proposes a prototype of virtual laboratory of mathematics to be working with this idea.
\end{abstract}

Keywords: teaching mathematics, problem solving, logic puzzle, computer resources in the teaching of mathematics. 


\section{Introdução}

Como um sistema lingüístico, a matemática utiliza-se de símbolos para comunicar significados. Assim, ao aprender matemática, já nas séries iniciais, o aluno se depara com uma linguagem formal.

Ao trabalhar com o ensino de matemática, o professor deve auxiliar o aluno na compreensão e na interpretação desta linguagem própria da matemática. Mas com o cuidado de reconhecer que o conhecimento matemático não é algo concluído e nem ter a idéia de que deve ser "transferido" por ele (que "conhece") ao aluno (que não possui ainda esse conhecimento organizado).

Segundo Vergnaud, a aquisição do conhecimento se dá, em geral, por meio de situações e problemas com os quais o aluno tem alguma familiaridade, o que implica em dizer que a origem do conhecimento tem características locais. "Um conceito não pode ser reduzido à sua definição, principalmente se nos interessamos por sua aprendizagem e seu ensino. É através das situações e dos problemas a resolver que um conceito adquire sentido para a criança" (Vergnaud 1993a).

Sendo assim, é importante que se use a resolução de problemas como uma metodologia de ensino em sala de aula. É uma atividade desafiadora, que faz com o que o aluno utilize sua criatividade e vá construindo os seus próprios conceitos e conhecimentos. Trabalhar com materiais instrucionais de um laboratório de matemática, pode ser uma maneira de trabalhar tal habilidade.

É comum ouvirmos relatos de muitos benefícios do uso do computador no processo de ensino-aprendizagem. É fácil observarmos o quanto determinados conteúdos de matemática são melhores compreendidos pelos alunos quando os conceitos são construídos a partir da imagem que um software matemático gerou ou quando se consegue visualizar imagens do que foi comentando em algum outro momento escolar. Também é clara a satisfação, a segurança que o aluno demonstra ao possuir um referencial de sua disciplina disponível a qualquer hora, que é o que acontece com o site da disciplina onde eles encontram todas as informações sobre o andamento da mesma (carga horária, dias e horários, sala, ementa, cronograma, materiais de apoio, exercícios, bibliografia, links interessantes, endereço de correio eletrônico do professor, site pessoal com informações do professor, etc). Sem contar que, cada vez mais os alunos estão habituados a buscarem um determinado assunto através de pesquisas na Internet.

Será que os benefícios que observamos do uso do computador no processo educacional não podem ser aliados aos benefícios de trabalharmos com materiais instrucionais e desafios de um laboratório de matemática? Afinal, a disponibilidade de usar um computador, muitas vezes, é algo mais acessível do que se ter um laboratório de matemática disponível para ser explorado. Isso acontece tanto no ambiente escolar quanto em outros locais onde o aluno possa estar. Com isso, pensamos em como adaptar materiais instrucionais e desafios tradicionalmente de um laboratório de matemática para atividades virtuais e trabalhá-los como ferramentas na resolução de problemas.

Pensando nisso, este artigo tem por objetivo abordar o ensino-aprendizagem de matemática, a resolução de problemas e apresentar um protótipo de laboratório virtual de matemática com tal proposta. $\mathrm{O}$ artigo está organizado da seguinte forma: a seção 
dois apresenta uma reflexão sobre o ensino-aprendizagem de matemática e levanta o uso das tecnologias computacionais em tal processo; a seção três trata da resolução de problemas, dando ênfase aos desafios; a seção quatro propõe um protótipo de laboratório virtual de matemática; e, finalmente, na seção cinco são apresentadas as considerações finais acerca deste trabalho.

\section{Refletindo sobre o processo de ensino-aprendizagem em matemática}

Podemos pensar na matemática como um alicerce fundamental na construção do conhecimento. Assim, ela precisa ser trabalhada de forma que se possibilite não só a aprendizagem dos conteúdos matemáticos, mas que se auxilie no desenvolvimento cognitivo do aluno como um todo. A resolução de problemas, o trabalho com desafios lógicos e com materiais concretos podem ser fundamentais para que se chegue a este nível. Quanto mais for trabalhada esta habilidade, mais aberto poderá ser o raciocínio do aluno e mais naturalmente poderá chegar a suas próprias conclusões.

Não muito distante, estávamos no tempo em que o professor só tinha como recursos didáticos o quadro-de-giz. Porém, atualmente, muitos outros recursos estão ao nosso alcance. Ao levarmos nosso aluno a um laboratório de materiais instrucionais de matemática, estamos dando a ele a chance de possuir uma maior interação, resolver problemas, enfrentar desafios, generalizar, compreender conceitos, etc., o que levará a construção do seu próprio conhecimento.

No entanto, a grande maioria das escolas, públicas ou privadas, não possui um laboratório de matemática para ser explorado pelos alunos. E, apesar de algumas universidades e escolas abrirem a visitação para outros alunos, isso já não é algo tão trivial a ser feito. E, quando é feito, acaba sendo algo esporádico, devido às dificuldades que envolvem tal trabalho diferenciado fora da escola.

Não é de hoje que se fala no uso do computador no processo de ensino aprendizagem. Atualmente, o computador vêm sendo bastante utilizado nesse processo educacional. Tal recurso já pode ser encontrado tanto em instituições de ensino particulares como em parte das escolas da rede pública de ensino.

Porém, assim como os recursos mais "tradicionais", como, por exemplo, livro, retroprojetor, vídeo, o computador não pode ser usado apenas para motivar os alunos e fazê-los participar mais ativamente do trabalho escolar. Como os outros recursos, ele deve ser um instrumento para comunicação de dados e que permita a interação entre estudantes e desses com seus professores. Esta inserção do computador no ensino é muito interessante para se propor trabalhos diferenciados com os alunos. Trata-se de um instrumento que pode ser utilizado de forma ativa e dinâmica, que fornece feedback, permitindo ao educando tentar novas alternativas e testar suas hipóteses.

No entanto, frisamos que não se trata de pensar que o computador venha a substituir o professor e tampouco que seja apenas mais um recurso para auxiliar na aprendizagem. $\mathrm{O}$ computador deve assumir um determinado papel na relação entre o aluno, o conhecimento e o professor. Ele é um recurso que utilizamos para atingir os objetivos educacionais propostos e melhorar a qualidade do ensino. Portanto, o emprego do computador no processo pedagógico, assim como o uso de qualquer tecnologia, exige do educador preparação e uma reflexão crítica. Surge assim a importância e a responsabilidade de nós, como professores, propormos esse tipo de atividades aos nossos alunos, sabendo escolhê-las e como direcioná-las no decorrer do trabalho. 


\section{Resolução de Problemas}

Diariamente, quando nos deparamos com uma situação nova, sobre a qual devemos pensar e usar todo o nosso conhecimento intelectual e habilidades para solucioná-la, estamos diante de um problema. Dentre estes problemas, estão os matemáticos. Devemos ter cuidado para não confundir um exercício matemático com um problema matemático. Enquanto que para resolvermos um exercício, usamos muito a repetição, para encontrarmos a sua solução de um problema matemático estamos sendo desafiados e precisamos usar estratégias e conhecimentos matemáticos. (Dante 1989)

Existem diversos tipos de problemas, bem como várias técnicas de resolução dos mesmos. Os desafios lógicos são um destes e podem ser trabalhados de maneira informal, sem ser em sala de aula, ou com orientação do professor, em sala de aula, adequando-os a cada série. Quem está habituado a trabalhar com desafios lógicos certamente terá a oportunidade de ter um pensamento mais amplo, não só para solucionar questões matemáticas.

\subsection{Alguns Marcos e Estudos em Resolução de Problemas}

No século passado, a resolução de problemas foi considerada como sendo uma aplicação do método de ensaio e erro, como associações entre estímulos e respostas, limitando-se a utilização mecânica de uma experiência anterior ou um acerto por acaso. Em 1925, Wolfgang Kohler, um dos psicólogos fundadores da Escola Gestalt, realizou uma pesquisa para descobrir como os macacos enjaulados faziam para pegar bananas que estavam fora da jaula. Assim, este pesquisador contradisse a descrição que vinha sendo dada para resolução de problemas e concluiu que os macacos reestruturavam o problema tendo um insight ${ }^{1}$. A partir daí, foram feitos estudos semelhantes para a resolução de problemas com humanos.

Muitas dessas idéias dos gestaltistas estavam vinculadas a Teoria do Significado. Essa teoria considera a existência de relações externas entre os dados do problema e os esquemas lógicos de quem o quer solucionar. Também defende que quando descobrimos por nós mesmos como solucionar o problema é bem mais produtivo. Mas, não houve grande interesse de estudos e pesquisas que comprovem esta idéia. Nesta área, um problema bastante conhecido é a Torre de Hanói, na qual temos 3 pinos e 64 discos com diferentes diâmetros; inicialmente, temos todos os discos em um dos pinos, em ordem decrescente, de baixo para cima. O desafio é montar a "torre" inicial em outro pino, movendo um disco de cada vez, sem colocar um disco maior sobre outro menor. Foram feitas adaptações com outras quantidades de discos. Vejamos a ilustração de uma dessas adaptações:

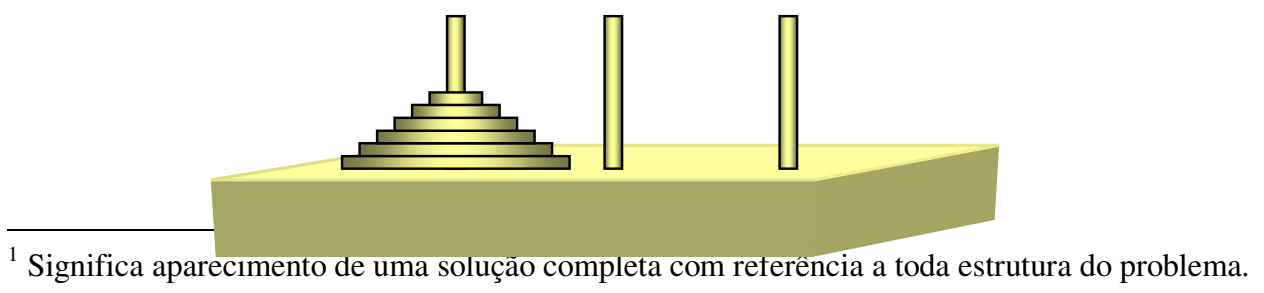


$\mathrm{Na}$ década de 50, com o progresso tecnológico, o avanço dos computadores, como máquinas que podem resolver problemas, começam realmente os estudos cognitivos. Nos anos 60, nos Estados Unidos, Bruner dirigiu o Projeto Cognição, considerando o sujeito como um solucionador de problemas ativo e construtivo, estudando suas estratégias mentais.

Seguindo os estudos, em 1972, Simon e Newell simulam o processo de compreensão do problema, separando-o em um sub-processo para interpretar a linguagem das instruções e outro para elaborar a representação mental do problema, com um programa de computador chamado Understand. Alguns anos depois, concluem que a representação que o sujeito constrói do problema está determinada pela forma como ele o entende. É importante lembrar que no processo de resolução de problemas, estão envolvidos fatores cognitivos, metacognitivos e afetivos, os quais estão relacionados com as experiências pessoais pelas quais o indivíduo passa. (Polya 1978)

\subsection{Estratégias de Resolução de Problemas}

Encontramos vários pesquisadores que se dedicam a estudar o assunto que está sendo tratado. Dentre esses, podemos destacar Polya [1978], um professor de Matemática que, utilizando a idéia gestáltica de reestruturação, propõe as seguintes etapas para se solucionar um problema:

\section{1) Compreender o problema}

Antes de tudo, o aluno precisa compreender o enunciado verbal; deve identificar as partes principais do problema, a incógnita, os dados e a condicionante. É fundamental que ele compreenda o problema e deseje resolvê-lo. Devemos estar atentos, pois se isto não ocorrer, pode ser sinal de que não escolhemos um bom problema, pois este deve ser nem muito difícil nem muito fácil, bem como natural e interessante.

\section{2) Estabelecer um plano}

O principal feito na resolução de um problema é a concepção da idéia de um plano. Para elaborar o plano, o aluno precisa saber quais os cálculos ou desenhos que serão utilizados para descobrir a incógnita. Ele deve perguntar-se se já solucionou anteriormente algum problema semelhante ao apresentado, se isso não ocorrer, será necessário que tente variar, transformar, reformular o problema, podendo chegar em um auxiliar se abandonar uma parte da condicionante, generalizar ou particularizar.

\section{3) Executar o plano}

Nesta etapa, o aluno irá colocar em prática o seu plano passo a passo, examinando detalhadamente cada um destes. Se o problema for muito complexo, podem ser distinguidos passos grandes e passos pequenos, corrigindo cada um destes de maneira formal ou intuitiva, sem deixar dúvidas de que está correto. Devem ser realizadas todas as operações algébricas ou geométricas que foram consideradas viáveis para a solução.

4) Fazer o retrospecto ou a verificação

É a etapa onde o aluno analisa a solução obtida, verifica o resultado e encontra possíveis enganos. Por isso, é muito importante e instrutiva para melhorar a resolução e aperfeiçoar a compreensão do problema. É interessante que seja feita uma discussão em 
grande grupo e se imagine casos em que pode ser usado o mesmo procedimento ou o resultado obtido, desta forma será percebida a relação que há entre problemas matemáticos.

Certamente estas etapas não precisam ser rigorosas ou seguidas à risca, mas nos orientam, segundo Polya, para chegarmos à solução.

\subsection{A Resolução de Problemas como Metodologia de Ensino}

Consideremos que qualquer situação onde ocorre um desequilíbrio fazendo com que usemos os nossos diversos conhecimentos, é um problema. Independente da série escolar, muitos alunos apresentam dificuldades para resolver problemas. Alguns pesquisadores dedicam-se a estudos que diminuam tal dificuldade.

Para Carvalho (1990), "não se aprende Matemática para resolver problemas e, sim, se aprende Matemática resolvendo problemas". Sendo assim, o professor deve propor em sala de aula tarefas interessantes que levem à questionamentos, que sejam ou que se transformem em problemas, criando um ambiente de pesquisa, de elaboração de estratégias, de soluções e de novos problemas. O professor precisa buscar meios de compreender o que se passa na sala de aula, os procedimentos dos alunos, as concepções que os mesmos têm, para planejar e propor problemas e desafios adequados (Vergnaud 1993b).

É importante ressaltar que o professor tem de estar preparado para aceitar diversos procedimentos dos alunos no decorrer da resolução, os quais podem ser diferentes daqueles que ele julga os melhores. Ele também deve propor novas representações que tornem os procedimentos dos alunos mais flexíveis e gerais, garantindo constantes discussões dos procedimentos em pequenos ou grandes grupos. Assim, há um geral enriquecimento espontâneo ou provocado pelo professor.

\subsection{Exemplos de desafios lógicos}

Os desafios lógicos também são problemas matemáticos. Alguns também podem envolver mais a álgebra ou a geometria. Vejamos alguns exemplos de desafios que podem ser trabalhados dentro ou fora da sala de aula.

\subsection{1 "Quadrado mágico"}

Como podemos colocar números de 1 à 9 , sem repeti-los, de modo que a soma de todas as linhas, colunas e diagonais sejam 15 ? (Figura 1)

Resolução - Lemos o enunciado buscamos entender o que queríamos descobrir. Então, pensamos ser o melhor colocar aleatoriamente os numerais de 1 à 9 nos quadradinhos, e fomos fazendo as somas e alterando as posições dos mesmos.

Podemos perceber que, para resolvermos, precisamos ler e compreender o enunciado. Sabido isso, tivemos que elaborar uma maneira de chegarmos onde queríamos. Assim, executamos o que planejamos e depois verificamos se deu certo. Portanto, segundo a interpretação acima, passamos pelas etapas descritas por Polya para resolver um problema. No momento em que precisamos usar o raciocínio lógico para descartar certos numerais em determinados quadrados, vimos que temos um desafio lógico. 
Então, na Figura 2 temos uma das soluções.

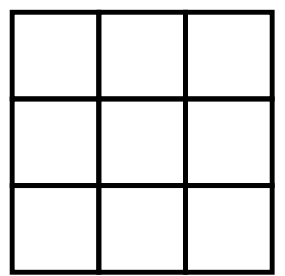

Figura 1 - Quadrado mágico

\begin{tabular}{|l|l|l|}
\hline 4 & 3 & 8 \\
\hline 9 & 5 & 1 \\
\hline 2 & 7 & 6 \\
\hline
\end{tabular}

Figura 2 - Solução do quadrado-mágico

\subsection{2 "Tangram"}

O Tangram (ou Tangran) é um quebra-cabeça chinês muito conhecido, de origem milenar. Segundo a lenda, o jogo surgiu quando um monge chinês deixou cair uma porcelana quadrada, que se partiu em sete pedaços - daí seu nome, que significa "tábua das sete sabedorias", "tábua das sete sutilezas", "as sete tábuas da argúcia". (Souza 1995)

É composto por sete peças (dois triângulos grandes; dois triângulos pequenos; um triângulo médio; um quadrado; um paralelogramo) resultantes da decomposição de um quadrado. Com estas peças é possível criar e montar inúmeras figuras, geométricas ou não.

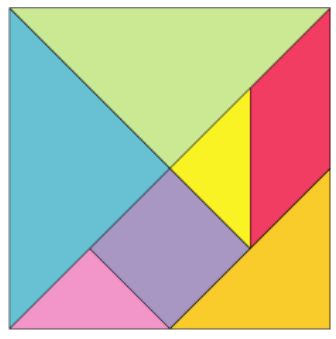

Figura 3 - Tangram

Ao trabalhar com desafios lógicos que envolvem geometria o aluno pode explorar formas geométricas, representá-las, compará-las, construí-las, etc. É uma maneira de desenvolver sua capacidade de visualização, de percepção espacial, de análise e criatividade. Através de desafios o aluno tem a oportunidade de construir conceitos matemáticos, empregar diversas maneiras para resolver problemas, ampliar suas habilidades em cálculo mental e em outros raciocínios lógico-matemáticos. E, segundo Vergnaud (1996), ao dar ao aluno a oportunidade de construir conhecimentos, cria-se melhores condições para a generalização de conhecimento.

\section{O Laboratório Virtual de Matemática}

Diante das questões inicialmente levantadas, realizamos um levantamento de materiais instrucionais e desafios existentes no laboratório de materiais instrucionais da Faculdade de Matemática da Pontifícia Universidade Católica do Rio Grande do Sul (FAMAT/PUCRS) e passamos a adaptá-los ao computador. Assim, estamos construindo um Laboratório Virtual de Matemática, tendo como proposta que ele seja 
utilizado por alunos de diferentes níveis escolares, em atividades direcionadas ou não por um professor.

Delimitando o escopo de nosso trabalho, o laboratório virtual que está sendo construído será explorado como parte do processo de desenvolvimento das aulas de matemática de uma escola regular. Para fins de pesquisa, coleta e análise de dados, estamos nos detendo a um público de dez alunos da $6^{\mathbf{a}}$ série do ensino fundamental, em uma escola privada de Porto Alegre. Essa escola possui seis turmas de $6^{a}$ série e cada uma delas tem, em média, trinta e cinco alunos, de onde serão selecionados aleatoriamente os participantes para a pesquisa.

Os dados estão sendo coletados através de: entrevistas individuais semiestruturadas com os alunos; observações estruturadas feitas dos alunos interagindo com o Laboratório Virtual; avaliações regulares que os alunos realizam em tal componente curricular; as quais são descritivas e avaliadas por objetivos, de acordo com os conteúdos que estão sendo trabalhados em sala-de-aula juntamente com o professor de tal componente curricular.

A pesquisa pode ser definida como de natureza qualitativa, já que a maior preocupação não será com a generalização dos resultados obtidos numa amostra, mas sim a caracterização, compreensão e interpretação dos fenômenos observados num grupo específico (Moura e Ferreira, 2005).

O site do Laboratório Virtual de Matemática é modelado no Microsoft Office FrontPage 2003 e pode ser acessado via web através do endereço http://www.ss.inf.br/labvirtual. Pretendemos colocar no site diferentes materiais que possam apoiar o aluno na construção do seu conhecimento matemático. Em um menu lateral, fizemos a seguinte divisão: Materiais, Desafios, Situações-problema.

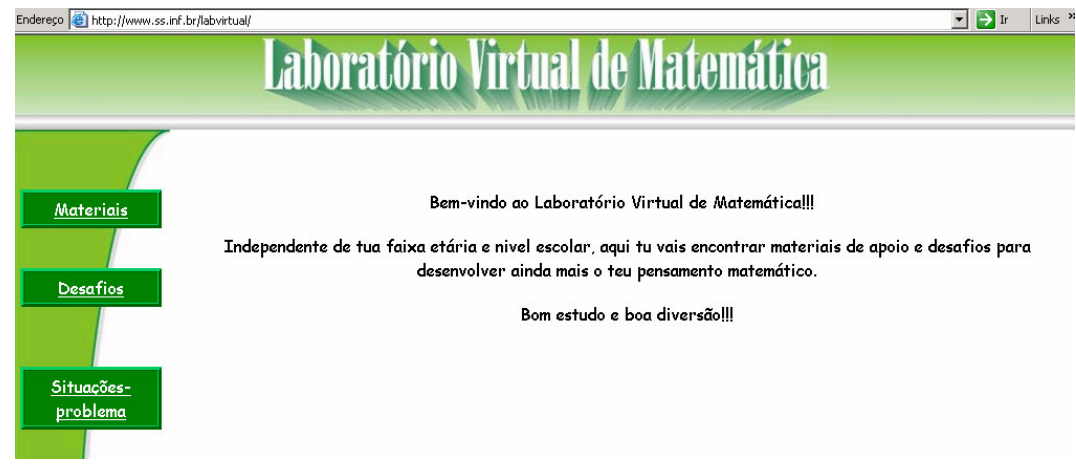

Figura 4 - Página inicial do Laboratório Virtual de Matemática

\subsection{Materiais}

Nesse local são colocados links para materiais de apoio sobre diferentes conteúdos que já foram ou estão sendo trabalhados com os alunos em sala de aula. Criamos um "sub-link" que aponta para um repositório de trabalhos desenvolvidos por alunos do curso de licenciatura em matemática, no qual a pesquisadora leciona. Esse repositório contém materiais de outros assuntos em matemática e, mesmo não sendo objeto de estudo dessa pesquisa, poderá ser utilizado como suporte para os alunos que forem trabalhar com os desafios. 


\subsection{Desafios}

Nesse espaço estão colocados os desafios lógicos. Por uma questão de organização, fizemos a seguinte divisão: desafios em Lógicos, Numéricos, Geométricos e colocamos link em cada um deles. No link Desafios Lógicos, estão os problemas clássicos de lógica. No link Desafios Numéricos, são encontrados os desafios lógicos que envolvem números. Nos Desafios Geométricos, estão aqueles desafios lógicos que envolvem figuras geométricas; um deles é o Tangram, o clássico quebra-cabeça chinês já citado aqui anteriormente. A grande maioria dos desafios numéricos e geométricos permitem que o usuário interaja com eles. Os desafios interativos são implementados usando o Macromedia Flash MX 2004.

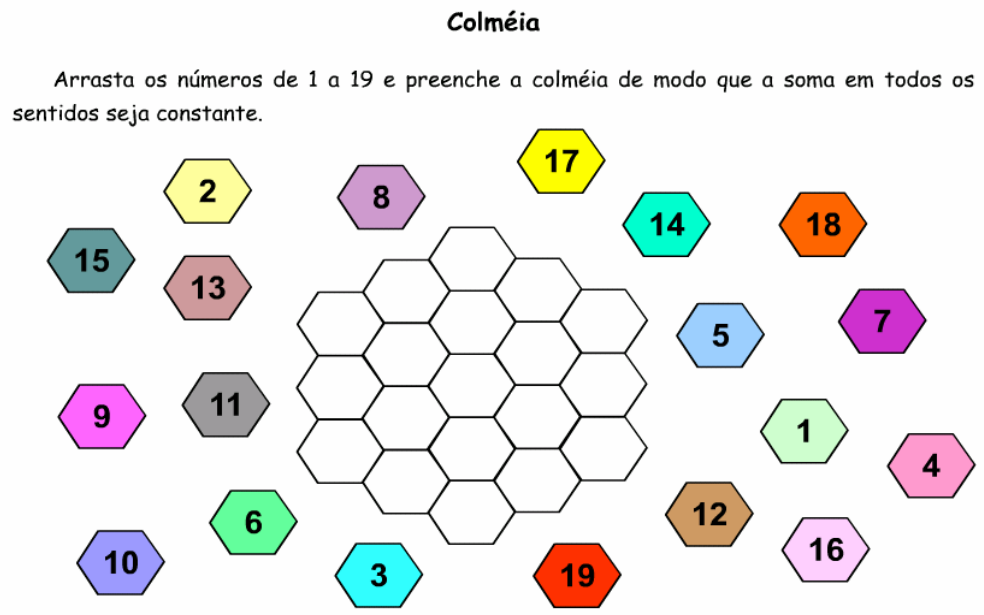

Figura 5 - Exemplo de Desafio Numérico.

\subsection{Situações-problema}

Assim como em "Materiais", aqui não é o espaço de maior exploração. São colocados links para situações sobre diferentes conteúdos que já foram ou estão sendo trabalhados com os alunos em sala de aula. Estão separadas em 3 grupos de acordo com os pré-requisitos mínimos necessários para a resolução (de acordo com os alunos da referida $6^{\mathrm{a}}$ série): Grupo 1, trabalhado desde ao $1^{\mathrm{o}}$ trimestre letivo; grupo 2, apenas após o final do $1^{\circ}$ e grupo 3, para o $3^{\circ}$ trimestre. São situações retiradas dos polígrafos desenvolvidos e trabalhados em sala de aula pela pesquisadora. Tal material constitui-se em complemento para quem estiver acessando o laboratório.

\section{Considerações Finais}

O professor de matemática possui responsabilidade com o ensino. Esta responsabilidade abrange um comprometimento com a ciência matemática, com a instituição na qual leciona, com a sociedade na qual vive e, principalmente, com o aluno. Em particular, o professor deve estar comprometido com a construção do conhecimento deste aluno. 
Fica evidente a necessidade de se pensar em uma aprendizagem da matemática, onde o aluno participa pensando sobre aquilo que faz, onde o conhecimento é construído ou reconstruído por ele mesmo, que traz implícito o desejo e a necessidade de conhecer. Dessa forma, o professor auxilia o aluno que, ao aprender matemática, não o faz de forma mecânica ou repetitiva como era feito há anos atrás em todas as escolas e, infelizmente, em algumas salas de aula dos dias de hoje ainda.

Não podemos pensar na matemática de maneira isolada. A matemática também deve contribuir para que o aluno desenvolva o seu modo de pensar e, com isso, uma maneira de agir, que lhe permita questionar sua vivência, criando e construindo ferramentas para resolver os problemas que forem se apresentando no decorrer de sua vida. Solucionar problemas faz parte do nosso dia-a-dia, mesmo que muitas não nos demos conta. Trabalhar com materiais instrucionais, resolução de problemas e desafios lógicos no ambiente escolar, ou fora dele, pode ser uma boa alternativa para desenvolver tal habilidade.

No entanto, na maioria das escolas aluno e professor não possuem acesso a um laboratório de matemática com tais materiais, o que dificulta tal processo. Porém, a disponibilidade de usar um computador com acesso a internet muitas vezes é algo viável. Isso acontece tanto no ambiente escolar quanto em outros locais onde o aluno possa estar. Sendo assim, com materiais instrucionais e desafios tradicionalmente de um laboratório de matemática adaptados para atividades virtuais e disponíveis via web podemos potencializar as alternativas que favoreçam a aprendizagem em matemática.

\section{Referências Bibliográficas}

CARVALHO, Dione Lucchesi de. Metodologia do ensino da matemática. 2. ed. São Paulo: Cortez, 1992.

DANTE, Luiz Roberto. Didática da resolução de problemas de matemática. São Paulo: Ática, 1989.

MOURA, Maria Lucia Seidl de; FERREIRA, Maria Cristina. Projetos de pesquisa: elaboração, redação e apresentação. Rio de janeiro: EDUERJ, 2005.

POLYA, George. A arte de resolver problemas. Rio de Janeiro: Interciência, 1978.

. O ensino por meio de problemas. Revista do Professor de Matemática, n. 7 , p. $11-16$, 2. sem. 1985.

SMOLE, Kátia C. S. (Org.). Ler, escrever e resolver problemas: habilidades básicas para aprender matemática. Porto Alegre: Artmed, 2001.

SOUZA, Eliane Reame et. al. A matemática das sete peças do tangram.São Paulo: Centro de Aperfeiçoamento do Ensino de Matemática, 1995. 102p.

VERGNAUD, Gérard. Teoria dos campos conceituais. In: Anais do $1^{\circ}$ Seminário Internacional de Educação Matemática do Rio de Janeiro. p. 1 - 26, 1993.

Piaget e Vygotsky - Convergências e controvérsias. In: Revista do GEEMPA. Porto Alegre, n. 2, p. 75 - 83, novembro 1993.

A trama dos campos conceituais na construção dos conhecimentos. In:

Revista do GEEMPA. Porto Alegre, n. 4, p. 9 - 19, julho 1996. 